\title{
Prospective Randomized Comparison between Sleeve Gastrectomy with Side-to-Side Jejunoileal Anastomosis and Sleeve Gastrectomy in the Treatment of Morbid Obesity
}

\author{
El-Sheikh M, MD; Hossam I, MD; Ismail T, MD; Abdel Hadey H, MD. \\ Department of General Surgery, Tanta University, Tanta, Egypt.
}

\begin{abstract}
Aim: The aim of the present research is to compare between sleeve gastrectomy with sideto-side jejunoileal anastomosis and sleeve gastrectomy in the treatment of morbid obesity in a prospective randomized manner.

Patiens and methods: This study was performed in General Surgery Department, Tanta University Hospitals, Egypt on 32 patients with morbid obesity. Patients were randomly categorized into 2 groups through a computer randomization program. Group J included 17 patients operated by sleeve gastrectomy plus a side-to-side jejunoileostomy. This group was compared to 15 patients undergoing conventional sleeve gastrectomy (Group S). The mean length of follow up was $22.4 \pm 5.68$ months in Group $J$ patients and $21.1 \pm 5.05$ months in Group S patients.
\end{abstract}

Results: For group J, mean operative time was $111.0 \pm 16.9$ versus $79.0 \pm 18.9$ minutes for group $S$ (p less than 0.0001). Mean hospitalization time was $5.35 \pm 1.50$ versus 3.73 \pm 0.884 days (p: 0.0010). Time to return to normal activity was $19.6 \pm 3.47$ and $18.1 \pm 3.28$ days (p 0.21). There was no mortality. For Group J, 3 patients (17.65\%) had superficial wound infection, 2 patients (11.76\%) developed postoperative atelectasis, 2 patients $(11.76 \%)$ developed gallbladder stones and1 patient (5.88\%) developed incisional hernia. Reversing the anastomosis was not necessary in any patient. For Group $S$, splenic injury occurred in 1 patient (6.67\%), 2 patients (13.33\%) had superficial wound infection, 1 patient (6.67\%) had deep wound infection with partial disruption, 2 patients (13.33\%) developed postoperative atelectasis, 1 patient (6.67\%) developed gallbladder stones and 1 patient (6.67\%) developed depression 6 months after surgery. Six months after operation, the mean BMI decreased by $23.35 \%$ and $12.89 \%$ ( $p: 0.058$ ). Twelve months after operation, the mean BMI decreased by $29.19 \%$ and $18.68 \%$ (p: 0.039). Two years after operation, the mean BMI decreased by $32.74 \%$ and $17.63 \%$ (p: 0.026). Mean duodenum to cecum transit time was $17.6 \pm 7.52$ versus $43.7 \pm 20.6$ minutes ( $p$ less than 0.0001). Seven patients with preoperative diabetes in Group J had normal fasting glucose in the first 3 postoperative months. Five (83.33\%) out 6 diabetic patients in Group $S$ discontinued all diabetic medication within the first 9 postoperative months. Four (80\%) out of 5 patients in group $J$ with preoperative hypertension showed complete disease resolution within the first 6 postoperative months, compared to $3(60 \%)$ out of 5 patients in the group $S$ over the same period of time. All of 9 patients (100\%) in Group $J$ with preoperative dyslipidemia had normal lipid profiles within the first 6 postoperative months. Only 3 out of 7 (42.86\%) patients from group $S$ had normal lipid profiles within the first 6 postoperative months and 5 out of 7 (71.43\%) at one year. Seven of the 8 patients in Group J with sleep apnea (87.5\%) and 7 out of 9 patients in Group $S$ (77.78\%) showed complete improvement 1 year after surgery. The rest showed partial improvement. All of the patients were satisfied with the results of the procedure. The mean patient satisfaction score was $9.12 \pm 0.781$ versus $8.80 \pm 0.775$ (p: 0.26).

Conclusions: The original design of sleeve gastrectomy with side-to-side jejunoileal anastomosis aims at adaptive and neuroendocrine goals as well as at restriction and malabsorption. Absence of prostheses or excluded segments, and easy feasibility associated with a metabolic corrective intervention in the context of adverse dietetic environments bring 
benefits to patients. Sleeve gastrectomy with side-to-side jejunoileal anastomosis may be a better procedure for the treatment of morbid obesity and an attractive alternative for the treatment of mildly obese patients with metabolic syndrome.

\section{Introduction:}

Obesity is one of the most important public health challenges of the 21st century: the World Health Organization has described it as an epidemic in developed nations and expressed concern about the cost of obesity to individual health and wellbeing, to health care systems, and economies generally. ${ }^{1}$

Overweight and obesity are the fifth leading risk for global deaths. At least 2.8 million adults die each year as a result of being overweight or obese. In addition, 44\% of the diabetes burden, $23 \%$ of the ischemic heart disease burden and between $7 \%$ and $41 \%$ of certain cancer burdens are attributable to overweight and obesity. ${ }^{2}$

Overweight and obesity lead to adverse metabolic effects on blood pressure, cholesterol, triglycerides and insulin resistance. Risks of coronary heart disease, ischemic stroke and type 2 diabetes mellitus increase steadily with increasing body mass index (BMI). Raised body mass index also increases the risk of cancer of the breast, colon, prostate, endometrium, kidney and gall bladder. Mortality rates increase with increasing degrees of overweight, as measured by body mass index. To achieve optimum health, the median body mass index for an adult population should be in the range of 21 to $23 \mathrm{~kg} / \mathrm{m} 2$. There is increased risk of co-morbidities for body mass index 25.0 to 29.9 , and moderate to severe risk of comorbidities for body mass index greater than $30 .^{2}$

As bariatric surgery evolves to metabolic surgery, there is a greater need for new surgical techniques that both promote substantial weight loss and improve metabolic comorbidities of obesity, such as diabetes mellitus. Because obese subjects constitute a unique group of surgical patients, these techniques also need to be simple and safe, with low morbidity and mortality.
A combination of sleeve gastrectomy and a side-to-side jejunoileal anastomosis wasintroduced to offer all of the known benefits of sleeve gastrectomy ${ }^{3,4}$ combined with enhanced neuroendocrine response from faster stimulation of the distal bowel, ${ }^{5-8}$ without excluding or resecting any segments of the digestive tract. Indeed, this operation is designed to combine the faster gastric emptying and the reduced ghrelin effect of sleeve gastrectomy ${ }^{3}$ with the shorter duodenum to cecum transit time associated with joining the proximal to the distal small bowel. This alteration of the gastrointestinal tract aims to change the bowel's response to food intake and enhance incretin release, which ultimately has beneficial effects onpancreatic beta cell function and generates satiety signals. ${ }^{5-8}$

The aim of the present research is to compare between sleeve gastrectomy with side-to-side jejunoilealanastomosis and sleeve gastrectomy in the treatment of morbid obesity in a prospective randomized manner.

\section{Patients and methods:}

This study was performed in General Surgery Department, Tanta University Hospitals, Egypt during the period from March 2011 to December 2013 on 32 patients with morbid obesity. Full explanation of procedures; possible complications and patient consent were assured before inclusion in the research. The study protocol was approved by the Ethics Committee of General Surgery Department, Tanta University Hospitals.

Patients were randomly categorized into 2 groups through a computer randomization program. Group J included 17 patients operated by sleeve gastrectomy plus a side-to- side jejunoileostomy. This group was compared to 15 patients undergoing conventional sleeve gastrectomy (Group S). 
All patients were operated on using an open approach. For Group J patients the sleeve gastrectomywas performed first, after using a sealer and divider device (LigaSure Atlas ${ }^{\circledR}$ ) to dissect the omentum away from the greater gastric curvature from approximately $5 \mathrm{~cm}$ proximal to the pylorus up to the angle of His. Excision of the fundus and the body of the stomach was then performed with linear stapler upon a 34 French orogastric tube (bougie). The gastric excision line was manually oversewn with a running suture. An isoperistaltic side-to-side anastomosis between the jejunum (at $100 \mathrm{~cm}$ distal to the Treitz ligament) and the distal ileum (at $100 \mathrm{~cm}$ proximal to the ileocecal valve) was then created using the same linear stapler. Stabilizing continuous bowel-to-bowel suturing with $2 / 0$ silk on each side of the anastomosis for a distance of approximately $10 \mathrm{~cm}$ was used to prevent twisting of the bowel and to reduce the possibility of mechanical obstruction. A tube drain was put at the left subdiaphragmatic space, and the abdominal wound was closed. For Group S patients the conventional Sleeve gastrectomy was performed.

One preoperative and 2 postoperative doses of Ceftriaxone sodium over $24 \mathrm{~h}$ were used for bacterial prophylaxis.

For deep venous thrombosis prophylaxis, elastic socks were utilized during the procedure and postoperatively until the patient was fully mobile. Subcutaneous Calciparine5,000 IU 2 hours before operation followed by 5,000 IU every 8 hours for 7 days or until the patient is ambulant.

Oral esomeprazole (40 mg daily) was also used for 1 month after discharge.

The patients were kept fasting for the first three postoperative days. On the fourth postoperative day, an upper GI series with gastrografin was performed to exclude gastric leaks and investigate the shape of the remaining stomach. When the gastrografin had advanced from the pylorus into the duodenum, serial films were taken at 5 minutes intervals, and the transit time from the duodenum to the cecum was recorded.

After the gastrografin test, the patients started a liquid diet for 1 week and then soft puree foods for three weeks. Multivitamins and high-protein liquid supplements were prescribed for the first 6 months. After this postoperative period, the patients were strongly advised to have small frequent meals, consuming mostly protein-enriched courses as well as fruit, vegetables, and water. Avoiding sugar, caloric liquids, and gas-producing liquids was recommended.

Patients were followed in visits at three day interval for two weeks then every 3 months. Patients that did not come for follow up were contacted by telephone. Patients were encouraged to visit the clinic at any time if they have any problem. The mean length of follow up was $22.4 \pm 5.68$ months in Group J patients and $21.1 \pm 5.05$ months in Group $\mathrm{S}$ patients.

\section{Statistical analysis}

Quantitative variables were expressed as mean $\pm \mathrm{SD}$. Qualitative variables were expressed as frequency and percent. Quantitative parametric variables were compared between the two groups using the unpaired student t-test, quantitative nonparametric variables were compared using Mann-Whitney test. Qualitative variables were compared using Chi-square test or Fisher exact test when the criteria for using Chi-square were not sufficient. The power used was 0.80 while the level of significance was $5 \%$.

\section{Results:}

The demographic characteristics of patients studied were summarized in Table (1).

In group J, 7 patients were diabetics; three had been using insulin for 1-7 years. There were 2 patients on oral anti-diabetic medication, while in 2 patients; the disease was diagnosed during the preoperative investigation. Hypertension (systolic blood pressure $>140 \mathrm{mmHg}$ and diastolic blood pressure $>90 \mathrm{mmHg}$ without medication) was present in 5 patients, dyslipidemia in 9 (cholesterol >200 mg/dl, Low-density lipoprotein $>130 \mathrm{mg} / \mathrm{dl}$, High-density lipoprotein $<40 \mathrm{mg} / \mathrm{dl}, \quad$ Triglycerides 
$>140 \mathrm{mg} / \mathrm{dl}$ ), and sleep apnea in 8 patients diagnosed by the sleep study.

In group S 6 patients were diabetics, 3 had been using insulin for $1-5$ years. There were 3 patients on oral anti-diabetic medication. Hypertension was present in 5 patients, dyslipidemia in 7 , and sleep apnea in 9 patients Table (1).

\section{Operation time:}

In group $\mathrm{J}$, operative time ranged from 90-150 minutes (mean operative time: 111.0 \pm 16.9 minutes). In group $\mathrm{S}$, operative time ranged from 60-120 minutes (mean operative time: $79.0 \pm 18.9$ minutes). The difference between the two groups was statistically significant (pless than 0.0001).

\section{Hospitalization time:}

Hospitalization time ranged from 3-10 days in both groups. Mean hospitalization time was $5.35 \pm 1.50$ days in group J. In group $\mathrm{S}$, mean hospitalization time was $3.73 \pm 0.884$ days. The difference between the two groups was statistically significant (p: 0.0010).

Time to return to normal activity:

Time to return to normal activity ranged from 14-30 days in group $\mathrm{J}$ (mean time: $19.6 \pm 3.47$ days). In group $\mathrm{S}$, time to return to normal activity ranged from 10-23 days (mean time: $18.1 \pm 3.28$ days). The difference between the two groups was statistically insignificant ( $p 0.21$ ).

\section{Complications}

For Group J patients there were no mortalities. Early postoperative complications appeared in five patients $(29.41 \%)$. Three patients $(17.65 \%)$ had superficial wound infection. They were treated with dressing and antibiotic. The other two patients $(11.76 \%)$ developed postoperative fever due to atelectasis and were treated conservatively with intensive respiratory physiotherapy. Two patients (11.76\%) developed gallbladder stones and underwent cholecystectomy. One patient $(5.88 \%)$ developed an incisional hernia. Reversing the anastomosis was not necessary in any patient.
For Group S patients there were no mortalities. Operative complications occurred in one patient (6.67\%). Splenic injury required splenectomy. Two patients (13.33\%) had superficial wound infection. They were treated with dressing and antibiotic. One patient $(6.67 \%)$ had deep wound infection with partial disruption. She was treated with antibiotics and secondary sutures. Two patients $(13.33 \%)$ developed atelectasis and were treated conservatively. One patient (6.67\%) developed gallbladder stones and underwent cholecystectomy. One patient $(6.67 \%)$ developed postoperative depression 6 months after surgery. He needed psychiatric help to improve.

Most of the patients reported no alterations in bowel movement frequency. Some patients reported episodes of diarrhea, especially after consuming fatty foods.There were no signs of malabsorption or malnutrition. No cases of dumping syndrome were observed Table (2).

\section{BMI study:}

Sixmonths after operation, the mean BMI decreased by $23.35 \%$ in group $\mathrm{J}$ and $12.89 \%$ in group $\mathrm{S}$ (p: 0.058). Twelve months after operation, the mean BMI decreased by $29.19 \%$ in group $\mathrm{J}$ and $18.68 \%$ in group $\mathrm{S}$ (p: 0.039 ). Two years after operation, the mean BMI decreased by $32.74 \%$ in group $\mathrm{J}$ and $17.63 \%$ in group S (p: 0.026). The results were statistically significantly better in group J than those observed after the sleeve gastrectomy alone at 6, 12 and 24 postoperative months Table (3).

Duodenum to cecum transit time:

In group J, duodenum to cecum transit time ranged from 5-30 minutes (mean duodenum to cecum transit time: $17.6 \pm 7.52$ minutes). In group $\mathrm{S}$, duodenum to cecum transit time ranged from 20-90 minutes (mean duodenum to cecum transit time: $43.7 \pm 20.6$ minutes). The difference between the two groups was statistically significant ( $p$ less than 0.0001).

Changes in preoperative comorbidities

All the 7 patients with preoperative diabetes in Group $\mathrm{J}$ discontinued all diabetic medications within the first three 


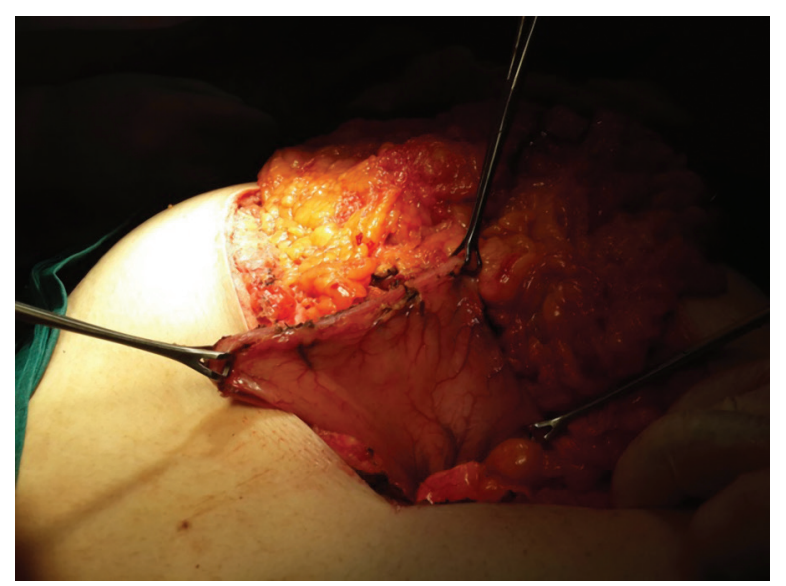

Figure (1): After devascularization of greater curvature.

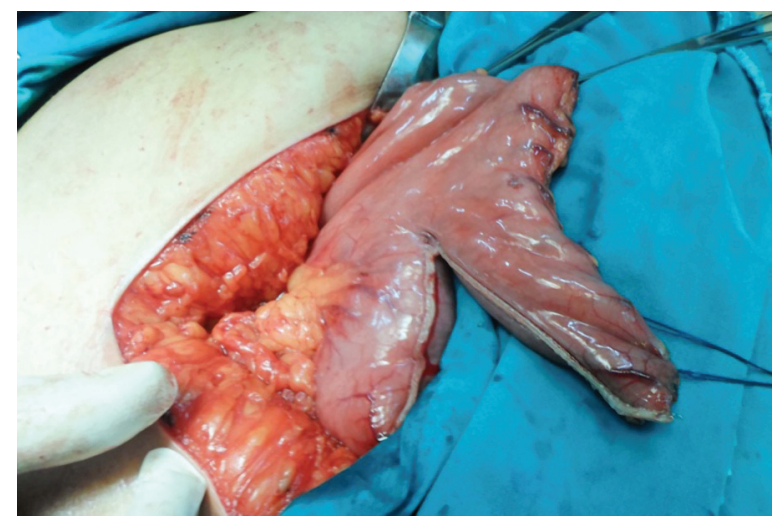

Figure (3): Excision of fundus and body of the stomach.

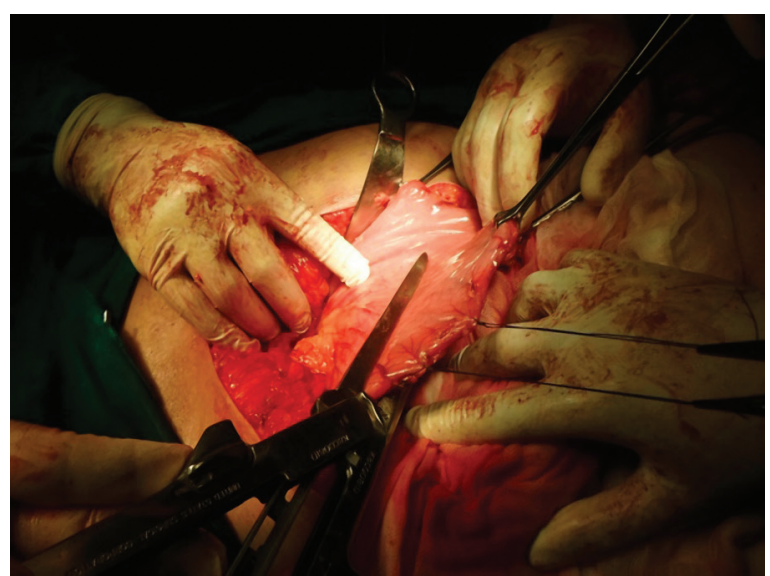

Figure (2): Application of linear stapler.

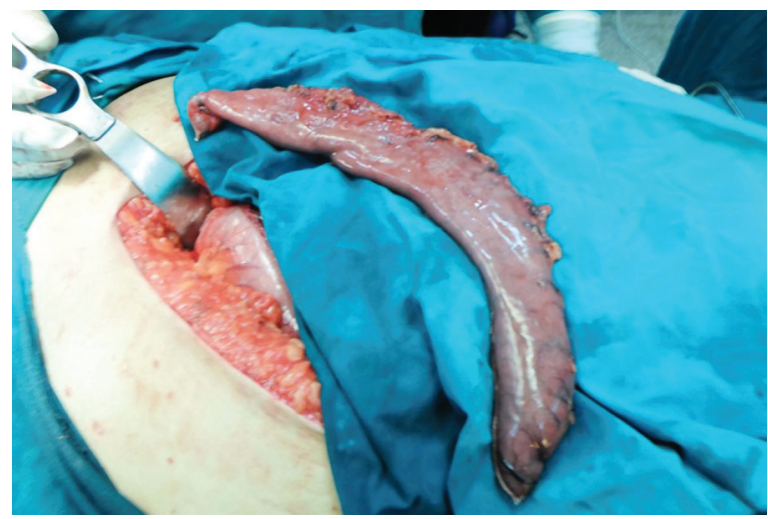

Figure (4): Complete sleeve gastrectomy.

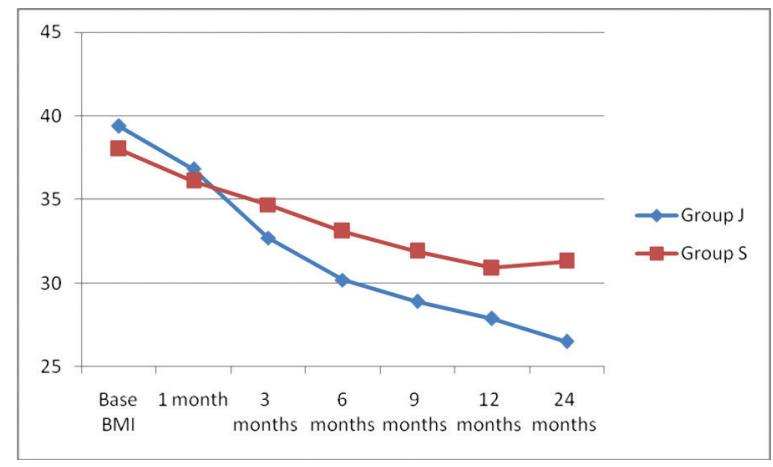

Figure (5): BMI changes in both groups.

postoperative months and had normal fasting glucose. Meanwhile, 5 (83.33\%) out 6 diabetic patients in Group S discontinued all diabetic medication within the first nine postoperative months. Until the end of follow-up, the status remained unchanged.

Four $(80 \%)$ out of 5 patients in group $\mathrm{J}$ with preoperative hypertension showed complete disease resolution within the first six postoperative months, as compared to $3(60 \%)$ out of 5 patients in the group $\mathrm{S}$ over the same period of time.

All of the nine patients (100\%) in Group $\mathrm{J}$ with preoperative dyslipidemia had normal lipid profiles within the first six postoperative months and were able to discontinue medical treatment. Onlythreeout of $7 \quad(42.86 \%)$ patients from group Shad normal lipid profiles within the first six postoperative months and 5 out of $7(71.43 \%)$ at one year were able to discontinue medical treatment.

All sleep apnea patients were followed 
Table 1: The demographic characteristics of patients.

\begin{tabular}{|l|l|l|l|}
\hline & Group J & Group S & $\mathrm{p}$ \\
\hline & N: 17 patients & N: 15 patients & \\
\hline Age & $38.9 \pm 12.1$ & $37.7 \pm 10.5$ & 0.78 \\
\hline $\begin{array}{l}\text { Gender } \\
\text { Males }\end{array}$ & 7 & 6 & \\
Females & 10 & 9 & \\
\hline Base BMI & $39.4 \pm 4.68$ & $38.0 \pm 4.90$ & 0.41 \\
\hline $\begin{array}{l}\text { Comorbidities } \\
\text { Diabetes }\end{array}$ & 7 patients & 6 patients & \\
Hypertension & 5 patients & 5 patients & \\
Dyslipidemia & 9 patients & 7 patients & \\
Sleep apnea & 8 patients & 9 patients & \\
\hline
\end{tabular}

Table 2 Postoperative complications in both groups

\begin{tabular}{|l|l|l|}
\hline complication & Number & Percentage \\
\hline Group J & & \\
Superficial wound infection & 3 & $17.65 \%$ \\
Atelectasis & 2 & $11.76 \%$ \\
Gallbladder stones & 2 & $11.76 \%$ \\
Incisional hernia. & 1 & $5.88 \%$ \\
\hline Group S & & \\
Splenic injury & 1 & $6.67 \%$ \\
Superficial wound infection. & 1 & $13.33 \%$ \\
Deep wound infection with partial disruption. & 1 & $6.67 \%$ \\
Atelectasis & 2 & $13.33 \%$ \\
Gallbladder stones (6.67 \%) & 1 & $6.67 \%$ \\
postoperative depression & 1 & $6.67 \%$ \\
\hline
\end{tabular}

Table 3: BMI changes inboth groups.

\begin{tabular}{|l|l|l|l|}
\hline & Group J & Group S & $\mathrm{p}$ \\
\hline Base BMI & $39.4 \pm 4.68$ & $38.0 \pm 4.90$ & 0.41 \\
\hline 1 month after operation & $36.8 \pm 4.74$ & $36.1 \pm 4.73$ & 0.71 \\
\hline 3 months after operation & $32.7 \pm 3.80$ & $34.7 \pm 4.37$ & 0.18 \\
\hline 6 months after operation & $30.2 \pm 3.87$ & $33.1 \pm 4.47$ & 0.058 \\
\hline 9 months after operation & $28.9 \pm 3.67$ & $31.9 \pm 4.29$ & 0.046 \\
\hline 12 months after operation & $27.9 \pm 3.51$ & $30.9 \pm 4.16$ & 0.039 \\
\hline 24 months after operation & $26.5 \pm 6.83$ & $31.3 \pm 4.01$ & 0.026 \\
\hline
\end{tabular}

postoperatively. Seven of the 8 patients in Group $\mathrm{J}$ with preoperative sleep apnea $(87.5 \%)$ and 7 out of 9 patients in Group S (77.78\%) showed complete improvement 1 year after surgery. The rest showed partial improvement.
Patient satisfaction

All of the patients were satisfied with the results of the procedure. The majority reported a significant reduction in total daily food intake and early satiety.Most of the patients reported alterations in their postoperative 
food preferences predominantly affecting sweets. The mean patient satisfaction score (Patient satisfaction score was designed by asking patients to express their satisfaction in a numerical score from 0 to 10 six months after surgery) for Group J patients was 9.12 \pm 0.781 versus $8.80 \pm 0.775$ for Group $S$ patients. The difference between the two groups was statistically insignificant (p: 0.26).

\section{Discussion:}

Humans learned to prepare food by selecting the richest parts of it and dismissing the low nutritive parts, as many other animals do, especially if there is abundance. However, very different from other animals, we started cooking, boiling, and liquefying food to make nutrients more available. Simply transforming apples into an apple puree causes significant changes in the patterns of absorption and glycemic response after a meal. ${ }^{9}$

Food industry developed new processes, such as refining, extrusion cooking, explosion puffing, utilizing extreme temperatures and pressure, or repeated wetting and drying of food. Such extremes facilitate the digestion of starches. ${ }^{10}$ These processes serve as external digestion. Frequently we are offered, with no effort or cost, completely digested food, such as pure glucose. In recent decades, such foods have become available on a daily basis, and they fully satisfy our most primitive instinct: obtaining nutritive food. The use of marketing aimed at reaching economic goals rather than nutritional goals worsens the medical problem.

Large meta-analyses showed that the bariatric procedures that work best (in terms of weight loss and metabolic improvement) are those that reduce the amount of food that is presented to the foregut and that enhance transportation of food to the hindgut. ${ }^{11}$

If a small segment of the proximal bowel is excluded, then good results still depend on some restriction, as in the Roux-en-Y Gastric Bypass. However, if a very long proximal segment is excluded, as in the biliopancreaticdiversion, then restriction is no longer needed for good metabolic results and weight loss, but malabsorption becomes a burden.

Natural selection confirms these findings, and the enrichment of a particular diet slowly leads to a selection of diminished stomachs and proximal guts. Carnivores have simpler and shorter guts than those animals that eat high-fiber, low-calorie diets. ${ }^{12}$ The progressive enrichment of diet during hominid evolution has also led to smaller abdominal cavities. ${ }^{13,14}$

Santoro et al. introduced the concept of a "neuroendocrine brake" as a means to digestive adaptation. They proposed some sort of a partial biliopancreatic diversion leaving $3 \mathrm{~m}$ of small bowel and without excluding the duodenum. ${ }^{15}$ Our approach attempts to exploit the relevant mechanisms of this proposal using a simpler and totally reversible procedure.

There are many aspects of current bariatric surgical procedures that should be avoided for a number of reasons. A purely restrictive procedure is less effective at controlling appetite, and the required dietary changes are usually hard to follow. Melissas et al. have recently shown that sleeve gastrectomy accelerates gastric emptying, thus proving that the procedure's mechanism of action involves more than simple restriction. ${ }^{4}$ This study was duplicated by other investigators with similar results. ${ }^{16,17}$ The alterations of the gastric motility following sleeve gastrectomyprobably cause alterations in the gut neuroendocrine mechanisms and result in early satiety and weight loss. ${ }^{18}$

Recently, a number of procedures that attempt to alter the intestines and affect the secretion of known neuroendocrine hormones have been introduced. According to our current knowledge and some research studies, $5,7,9,10$ bringing food in contact with the distal ileum earlier in the digestive process leads to secretion of incretins (GLP-1, PYY, $\mathrm{PP})$ that initiate a number of neuroendocrine changes, thus leading to earlier satiety and better regulation of energy and glucose homeostasis and ultimately to substantial weight loss. $5,7,8$

A side-to-side jejunoileal anastomosis is believed to be an easy, simple, considerably 
safe, and effective method for enhancing the neuroendocrine response of the intestine to food consumption. It is thought to suppress orexigenic pathways and lead to prompt satiety due to earlier contact between ingested food and the distal ileum without any blind small bowel loops.

Sleeve gastrectomy with side-to-side jejunoileal anastomosis seems to have metabolic and weight loss effects similar to those observed with biliopancreatic diversion and the ileal interposition, but with less operative risk due to reduced number of anastomoses. These qualities make it a possible candidate operation after failed sleeve gastrectomy or in patients with T2DM over 5 years.

\section{Conclusion:}

In conclusion, the original design of sleeve gastrectomy with side-to-side jejunoileal anastomosis aims at adaptive and neuroendocrine goals as well as at restriction and malabsorption. Absence of prostheses or excluded segments, and easy feasibility associated with a metabolic corrective intervention in the context of adverse dietetic environments bring benefits to patients. Sleeve gastrectomy with side-to-side jejunoileal anastomosis may be a better procedure for the treatmentof morbid obesity and an attractive alternative for the treatment of mildly obese patients with metabolic syndrome.

\section{Reference:}

1- Surgery for morbid obesity: Framework for bariatric surgery in Victoria's public hospitals, www.health.vic.gov.au, March 2009

2- WHO,Obesity and overweight, Fact sheet N³11 http://www.who.int/mediacentre/ factsheets/fs311/en/ last updated March 2013

3- Aggarwal S, Kini SU, Herron DM: Laparoscopic sleeve gastrectomy for morbid obesity: A review. Surg Obes Relat Dis 2007; 3: 189-194.

4- Melissas J, Koukouraki S, Askoxylakis J, etal. Sleeve gastrectomy: A restrictive procedure? Obes Surg 2007; 17: 57-62.

5- Murphy KG, Dhillo WS, Bloom SR: Gut peptides in the regulation of food intake and energy homeostasis. Endocr Rev. 2006; 27: 719-727.
6- Gallgher TK, Geoghegan JG, Baird AW, et al: Implications of altered gastrointestinal motility in obesity. Obes Surg. 2007; 17: 1399-1407.

7- Rubino F, Zizzari P, Tomasetto C, et al: The role of the small bowel in the regulation of circulatin ghrelin levels and food intake in the obese Zucker rat. Endocrinology 2005; 16: 1745-1751.

8- Bose M, Olivan B, Teixeira J, et al: Do incretins play a role in the remission of type 2 diabetes after gastric bypass surgery: What are the evidence? Obes Surg. 2009; 19: 217-229.

9- Haber GB, Heaton KW, Murphy D, et al: Depletion and disruption of fiber: effect on satiety, plasma-glucose and serum insulin. Lancet 1977; 2: 679-682.

10- Brand CJ, Nicholson PL, Thornburn AW, et al: Food processing and glycemic index. $\mathrm{Am}$ J Clin Nutr 1985; 42: 1192-1196.

11- Montgomery IA, Irwin N, Flatt PR: Active immunization against (Pro(3)) GIP improves metabolic status in high-fat-fed mice. Diabetes Obes Metab 2010; 12: 744-751.

12- Buchwald H, Avidor Y, Braunwald E, et al: Bariatric surgery: A systematic review and meta-analysis. JAMA 2004; 292: 1724-1737.

13- Leonard WR, Robertson ML: Evolutionary perspectives on human nutrition: The influence of brain and body size on diet and metabolism. Am J Hum Biol1994; 6: 77-88.

14- Aiello LC, Wheeler P. The expensive tissue hypothesis: The brain and the digestive system in human and primate evolution. Curr Anthropol 1995; 36: 199-221.

15- Santoro S, Castro LC, Velhote MC, Malzoni CE, Klajner S, Castro LP, Lacombe A, Santo MA: Sleeve gastrectomy with transit bipartition: A potent intervention for metabolic syndrome and obesity. Ann Surg 2012; 256(1):104-110.

16- Braghetto I, Davanzo C, Korn O, et al: Scintigraphic evaluation of gastric emptying in obese patients submitted to sleeve gastrectomy compared to normal subjects. Obes Surg 2009; 19: 1515-1521.

17- Melissas J, Daskalakis M, Koukouraki S, et al: Sleevegastrectomy: A "food limiting" operation. Obes Surg 2008; 18: 1251-1256.

18- le Roux CW, Aylwin SJB, Batterham RL, et al: Gut hormone profiles following bariatric surgery favor an anorectic state, facilitate weight loss and improve metabolic parameters. Ann Surg 2006; 243: 108-114. 